\title{
DIAGNÓSTICO DE BOVINOS LEITEIROS EM DUAS PROPRIEDADES DE MATO GROSSO
}

\author{
DIAGNOSIS OF DAIRY CATTLE IN TWO SMALL PROPERTIES OF MATO \\ GROSSO
}

\author{
Glaucia Folador Rech ${ }^{1}$ (D), Wesley Bonfim da Silva Borges ${ }^{2}$ (D), Eudineia Pinto Maciel $^{3}$ (D) \\ Poliana Alves Damacena ${ }^{4}$ (D) Késia Prates Meneghini ${ }^{5}$ (D)
}

Recebido em 29 de Setembro de 2020 | Aprovado em 17 de Dezembro de 2020.

\section{RESUMO}

O leite é um dos produtos mais consumidos pela sociedade em todo o mundo, e esse consumo vem crescendo gradativamente no Brasil. Diante disto, a produção de leite é uma alternativa que pode ser muito rentável para os produtores e para a cadeia produtiva. Sabendo da necessidade dos produtores rurais em obter práticas eficientes para a produção, objetivou-se conhecer o processo de criação de gado leiteiro em uma propriedade rural localizada no município de Vila Rica e outra em Canabrava do Norte (MT). Foram analisadas as seguintes características: manejo, período de lactação, quadro reprodutivo e escore corporal de animais nessas propriedades. Para a produção dos dados utilizamos estudos bibliográficos em sites e artigos, além de pesquisa de campo. Os procedimentos utilizados foram questionários, observações e manuscritos. Ao analisar os resultados obtidos verificou-se que o acompanhamento técnico dessas propriedades é de suma importância para o produtor, visando garantir a boa produtividade dos animais. Percebe-se, ainda, que a propriedade que teve maior persistência no programa Nosso Leite da Embrapa (Empresa Brasileira de Pesquisa Agropecuária), obteve maior êxito em sua produtividade comparando-se com a outra propriedade.

Palavras-chave: Controle da produção; Gado leiteiro; Manejo dos animais; Pequenos produtores.

\begin{abstract}
Milk is one of the most consumed products by society worldwide, and this consumption has been growing gradually in Brazil. Before this, milk production is an alternative that can be very profitable for producers and for the production chain. Knowing the need of rural producers to obtain efficient production practices, the objective was to learn about the process of raising dairy cattle on a rural property located in the municipality of Vila Rica and another in Canabrava do Norte (MT). The following characteristics were analyzed: management, lactation period, reproductive status and body score of animals in these properties. For the production of the

\footnotetext{
${ }^{1}$ Universidade do Estado de Mato Grosso- UNEMAT Vila Rica. Endereço para correspondência: Av. Perimetral Norte, 422-582, Vila Rica - MT, 78645-000. E-mail: glauciafoladorrech@ hotmail.com

${ }^{2}$ Universidade do Estado de Mato Grosso- UNEMAT Vila Rica. Endereço para correspondência: Av. Perimetral Norte, 422-582, Vila Rica - MT, 78645-000. E-mail: bonfimdasilvaborges@gmail.com

${ }^{3}$ Universidade do Estado de Mato Grosso- UNEMAT Vila Rica. Endereço para correspondência: Av. Perimetral Norte, 422-582, Vila Rica - MT, 78645-000. E-mail: eudyneiamaciel@gmail.com

${ }^{4}$ Universidade do Estado de Mato Grosso- UNEMAT Vila Rica. Endereço para correspondência: Av. Perimetral Norte, 422-582, Vila Rica - MT, 78645-000. E-mail: damacenapoliana@gmail.com

${ }^{5}$ Universidade do Estado de Mato Grosso- UNEMAT Vila Rica. Endereço para correspondência: Av. Perimetral Norte, 422-582, Vila Rica - MT, 78645-000. E-mail: kesiaprates@ outlook.com
} 
data we use bibliographic studies on websites and articles in addition to field research. The procedures used were questionnaires, observations and manuscripts. When analyzing the results obtained, it was found that the technical monitoring of these properties is of paramount importance for the producer, aiming to guarantee the good productivity of the animals. It is also noticed that the property that had greater persistence in the Nosso Leite program, of the Embrapa (Empresa Brasileira de Pesquisa Agropecuária), was more successful in its productivity compared to the other property.

Keywords: Production control; Dairy cattle; Animal management; Small producers.

\section{Introdução}

A produção de leite é de suma importância para a humanidade, pois este é um dos alimentos mais consumidos no mundo. O Instituto Brasileiro de Geografia e Estatística (IBGE) investiga a produção desse alimento no Brasil desde o ano de 1976. Esses estudos indicam um crescimento de mais de 3 milhões de litros na produção de leite cru de de 1997 até o segundo semestre de 2019, totalizando 5.854.269 milhões de litros de leite cru produzidos ao ano (IBGE, 2019).

Um fator que exerce forte influência na produção de leite é o escore corporal das vacas em lactação. Ele deve ser avaliado sempre que possível, para garantir reservas energéticas suficientes para o animal. Segundo Lago e colaboradores (2001, p. 01) "A medida do Escore de Condição Corporal (ECC) é uma maneira subjetiva de se avaliar as reservas energéticas da vaca, sendo baseado na observação visual e palpação de áreas específicas para avaliar os depósitos de tecido adiposo e massa muscular". As mudanças no ECC podem prejudicar os períodos que antecedem a reprodução dos animais. Assim, as vacas quando estão prenhas devem ser bem avaliadas e cuidadas, pois na escala de 1 a 5 de ECC o valor mais adequado é a 3 . Se o escore for menor, as vacas tendem a desenvolver mais doenças. Se o escore corporal for muito alto também pode ocasionar vários problemas de saúde.

Outro aspecto importante quanto ao ECC é sua variação, ou seja, deve-se evitar mudanças bruscas no período de transição das vacas (30 dias pré e pós parto), pois isso prejudica o desempenho produtivo e reprodutivo, podendo debilitar o animal ou leva-lo a morte. A avaliação do ECC de vacas prenhas pode evitar que estas sofram com falta ou excesso de peso durante o parto (LAGO et al., 2001).

Outro fator observado em fazendas produtoras de leite é o intervalo entre partos de uma mesma vaca. Este intervalo corresponde ao período de serviço que deve ser no máximo de 90 dias, para se obter um intervalo de partos de 12 meses. Garantindo, assim, que a cada ano uma mesma vaca tenha uma gestação, aumentando a eficiência da produtividade do rebanho (FERREIRA; MIRANDA, 2007; GOMES, 2018). 
Os intervalos entre os partos também são de extrema importância pra a saúde animal, sendo necessário que o produtor forneça o período de descanso de 60 dias antes do parto às vacas. Caso isso não aconteça o produtor poderá ter grandes prejuízos, uma vez que seu animal poderá adoecer ou até mesmo morrer durante o parto. Sem o devido descanso a vaca não conseguirá produzir o colostro, alimento necessário para a saúde do animal rebento.

Observa-se também, em algumas propriedades, o manejo na fase do período de lactação. Para que haja uma boa produção de leite vários fatores devem ser levados em consideração, tais como: raça, genética, fatores ambientais e, principalmente, a alimentação. Outro fator de grande importância na produção leiteira é a idade de produção da vaca, pois se a mesma não está fornecendo uma boa produção, ou seja, não está produzindo uma quantidade satisfatória de leite, a mesma deverá ser descartada e destinada ao abate (CERDÓTES, 2004).

Alguns parâmetros são utilizados para estimar a curva de lactação de um rebanho, tais como: tempo de pico, pico na produção e persistência. Tempo de pico é o período em que o animal está produzindo leite. O pico de produção é o momento em que a produção está em alta, e a persistência se define quando o animal mantem o pico de produção em alta (CRUZ, 2009).

Para facilitar o produtor na organização e no manejo do gado leiteiro, a Embrapa desenvolveu o quadro reprodutivo que tem o intuito de melhorar o rendimento do rebanho, permitindo ao produtor acompanhar o desenvolvimento de cada animal. Segundo Bergamaschi et al. (2010), é preciso manter a organização e o controle da reprodução de um rebanho a fim de identificar possíveis situações que estejam causando perdas visando resolvê-las antes de maiores prejuízos. O controle reprodutivo permite, ainda, otimizar a produção aproveitando melhor as instalações, o capital investido, a mão-de-obra, a genética dos animais, dentre outros fatores (BERGAMASCHI et al., 2010).

De acordo com dados da Embrapa (VILELA et al., 2016) cerca de 90\% dos produtores que estão inseridos no Programa Nosso Leite possuem o quadro reprodutivo em suas propriedades. O quadro reprodutivo é uma roda física onde "o animal é representado por um imã colorido, posicionado e movido de acordo com a fase reprodutiva em que se encontra" (ROSSO; RODRIGUEZ, 2016). Assim sendo, o registro das atividades na propriedade deve ter um controle diário, como por exemplo, entrada no cio, coberturas, partos, medicamentos ou vacinações, doenças e outros.

Percebendo a necessidade dos produtores em aperfeiçoar seus trabalhos com o gado leiteiro, este estudo buscou entender a rotina de duas propriedades mato-grossenses, uma localizada em Canabrava do Norte e outra em Vila Rica. Sabe-se que muitos proprietários, que desempenham atividade leiteira, já aderiram aos métodos ou técnicas a fim de melhorar o 
desempenho de suas propriedades. Contudo, alguns produtores de leite ainda têm dificuldade em aceitar a mudança ou se inteirar das técnicas. Para ajudar a derrubar este paradigma tanto os estudos de caso como cursos ou programas de extensão podem auxiliar aos envolvidos.

\section{Metodologia}

Em julho de 2019 foi realizado o diagnóstico na fazenda Estância do Ouro localizada a 500 metros da cidade de Canabrava do Norte - MT (Coordenadas: latitude da sede 419427; longitude 8769418; latitude do curral 419466; longitude 8769483). O produtor mora há dez anos nesta propriedade e desde o ano 2010 trabalha com o gado leiteiro. Este produtor aderiu ao Projeto Nosso Leite, utilizando 1,3 hectare (ha) de pasto no início e dispondo de 23 ha, atualmente, para as pastagens. Na Figura 1 é demonstrada uma parte do rebanho de produção leiteira da propriedade Estância do Ouro.

Figura 1 - Rebanho leiteiro na propriedade Estância do Ouro em Canabrava do Norte - MT.

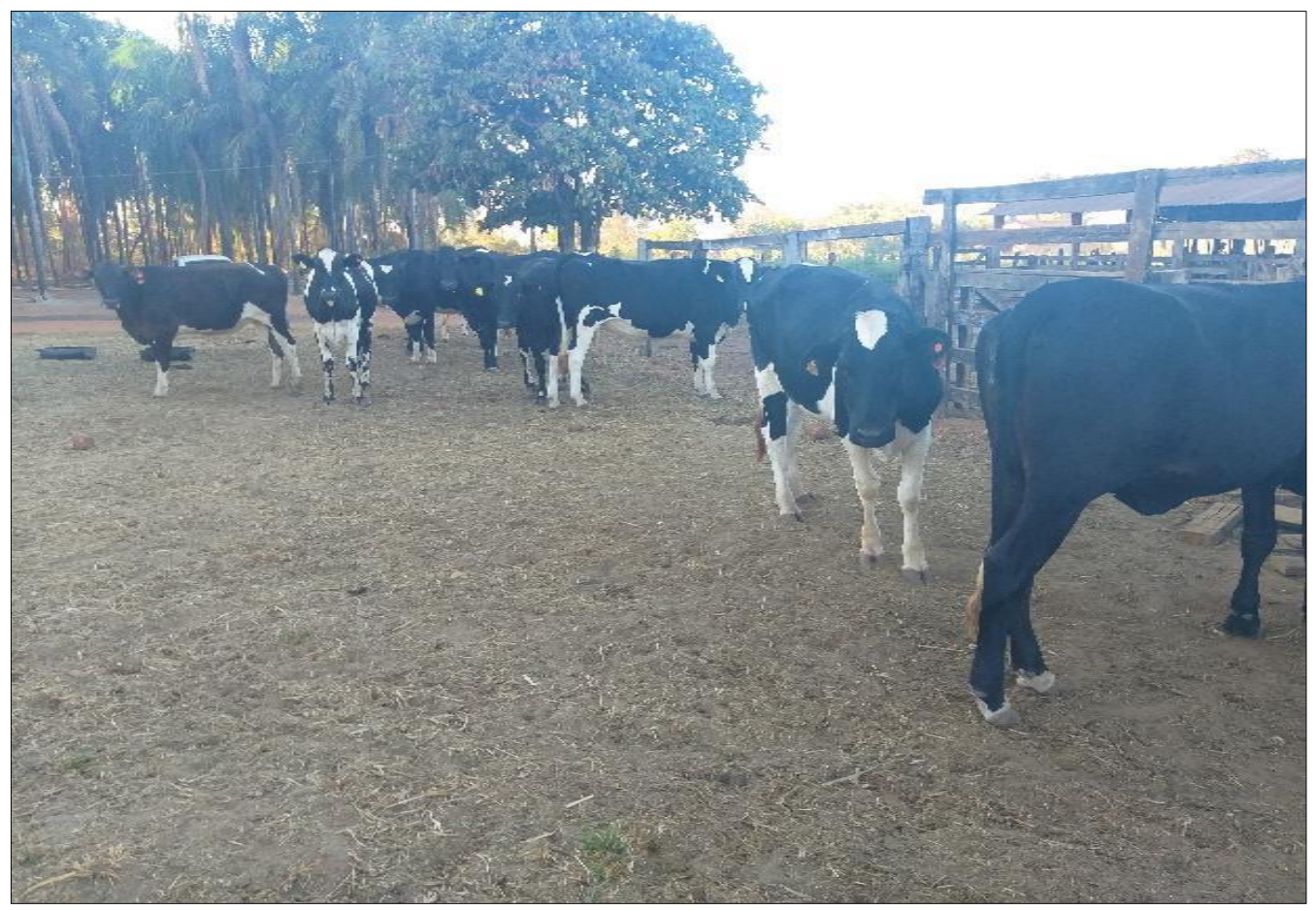

Fonte: Obtido pelos autores (2019).

Já na Tabela 1 é possível realizar um paralelo entre as propriedades Estância do Ouro (Canabrava do Norte) e Laço de Ouro (Vila Rica) no que se refere ao tamanho da propriedade, área destinada ao pasto (\%), tamanho do rebanho e distribuição dos animais (\%). 
Tabela 1 - Comparações entre as propriedades Estância do Ouro (Canabrava do Norte/MT) e Sítio Laço do Ouro (Vila Rica/MT). Fonte: Obtido pelos autores (2019).

\begin{tabular}{rcc}
\hline \multirow{2}{*}{ Descrição } & \multicolumn{2}{c}{ Propriedades } \\
& Estância do Ouro & Sítio Laço do Ouro \\
\hline Tamanho da propriedade (ha) & 48 & 52 \\
Área destinada à pastagem (\%) & 48 & $50^{*}$ \\
Rebanho Total & 64 & 62 \\
Vacas em lactação (\%) & 37 & 34 \\
Vacas Prenhas (\%) & 6 & 13 \\
Novilhas inseminadas (\%) & 28 & 5 \\
$\operatorname{Outros}^{* * *}(\%)$ & 29 & 48 \\
\hline
\end{tabular}

\footnotetext{
${ }^{*}$ Valor estimado informado pelo proprietário. ${ }^{* *}$ Bezerros - machos / fêmeas (1 a 24 meses) e bois.
}

A cobertura de todo o rebanho na propriedade Estância do Ouro é feita por inseminação artificial, no ato da inseminação verifica-se a avaliação do ECC de cada vaca. O produtor realiza a retirada do leite duas vezes ao dia, pelo método de ordenha mecânica (Figura 2), totalizando uma média diária de 340 litros. Uma parte do leite produzido é comercializado em uma empresa de laticínios e a outra é destinada aos bezerros (ainda em fase de amamentação). O valor recebido por cada litro de leite é de 1,21 reais. A alimentação do gado ocorre por meio de pasto rotacionado, na época das águas, e no período seco é oferecido cana de açúcar triturada e capim (produzidos pelo sistema de irrigação implantado na propriedade).

Figura 2 - Sala de ordenha na propriedade Estância do Ouro em Canabrava do Norte/MT.

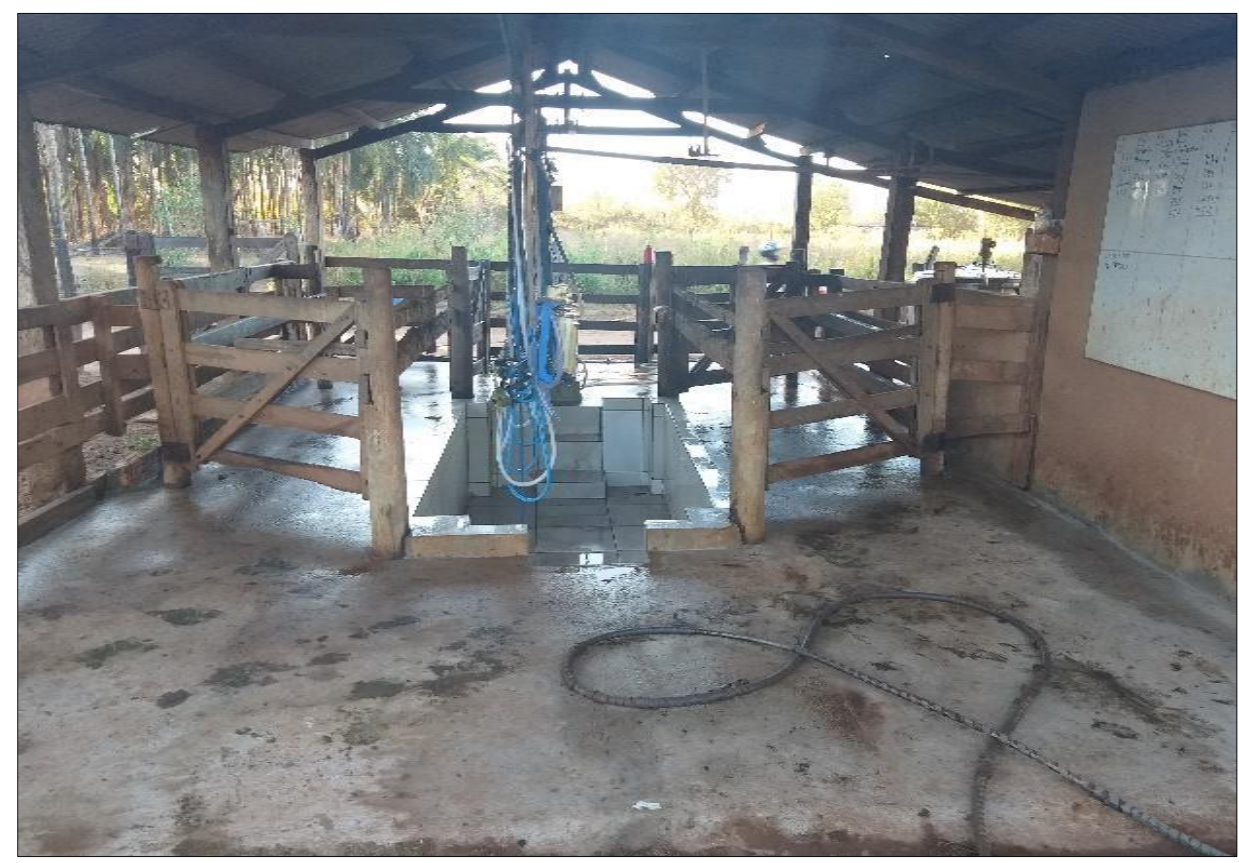

Fonte: Obtido pelos autores (2019). 
$\mathrm{Na}$ referida propriedade o produtor usa o quadro reprodutivo desenvolvido pela Embrapa para auxiliar no manejo produtivo e de reprodução dos animais. Neste quadro reprodutivo, demonstrado na Figura 3, percebe-se que é possível conhecer a propriedade totalmente, verificando assim a quantidade de animais bem como o período funcional no qual eles se encontram.

Figura 3 - Quadro de Controle de reprodução da propriedade Estância do Ouro.

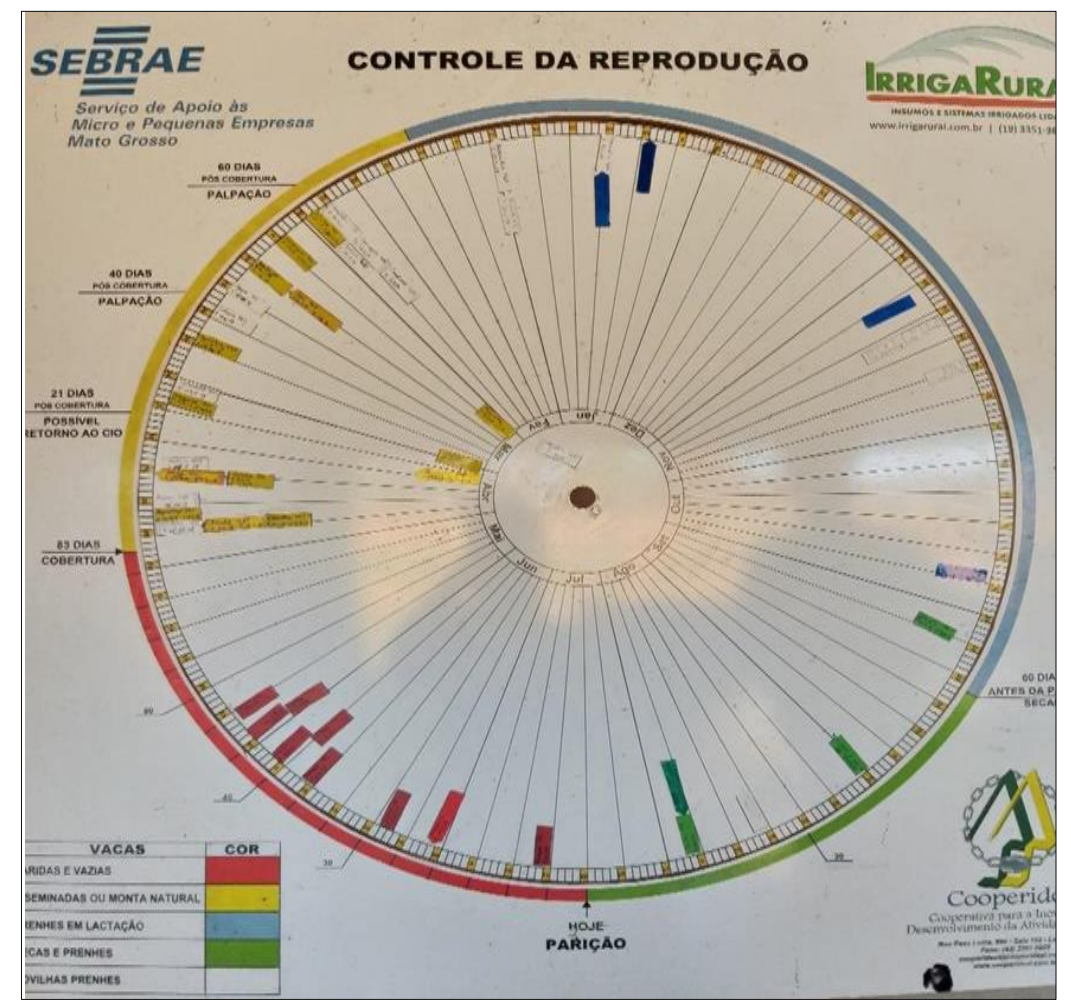

Fonte: Obtido pelos autores (2019).

A outra propriedade avaliada foi o Sítio Laço do Ouro em Vila Rica - MT, a $20 \mathrm{~km}$ de distância da área urbana do município. O diagnóstico desta propriedade também foi realizado em julho de 2019. Atualmente, o proprietário do Sítio Laço de Ouro não está inserido no Projeto Nosso Leite, mas participou durante três anos do projeto Balde Cheio (projeto também criado pela Embrapa que deu origem ao projeto Nosso Leite. Este produtor desistiu do projeto devido à falta de persistência e as exigências impostas pelo projeto.

Nesta propriedade a alimentação dos animais é feita a pasto extensivo e com sal mineral no período das chuvas. No período da seca a alimentação fornecida é sal mineral e cana de açúcar triturada. O produtor mora neste sítio desde 2008 e esta adequando os pastos para o manejo rotacionado. Sua renda familiar mensal é retirada desta propriedade com a venda dos leite, bem como de derivados, e outros animais de criação. Contudo, não é feito um controle 
financeiro do benefício-custo da propriedade.

A quantidade de animais, bem como a distribuição destes, está descrita na Tabela 1 onde se compara esta propriedade com a outra, Estância do Ouro em Canabrava do Norte - MT. A cobertura de todo o rebanho do Sítio Laço do Ouro (Vila Rica - MT) é feita por monta natural. A observação e avaliação do ECC das vacas não é realizado com frequência e, portanto, não se tem um controle efetivo.

A Figura 4 demonstra uma parte do gado em pastagem extensiva na propriedade Laço do Ouro. Boa parte da produção do leite é vendida, outra parte é utilizada para consumo próprio ou destinada aos bezerros que ainda estão na fase de amamentação. O valor recebido por cada litro de leite vendido é de 1,04 reais, sendo feita duas ordenhas ao dia totalizando uma quantidade diária de 96 litros de leite. Toda a ordenha é feita manualmente.

Figura 4 - Vaca e bezerros na propriedade Laço do Ouro em Vila Rica - MT.

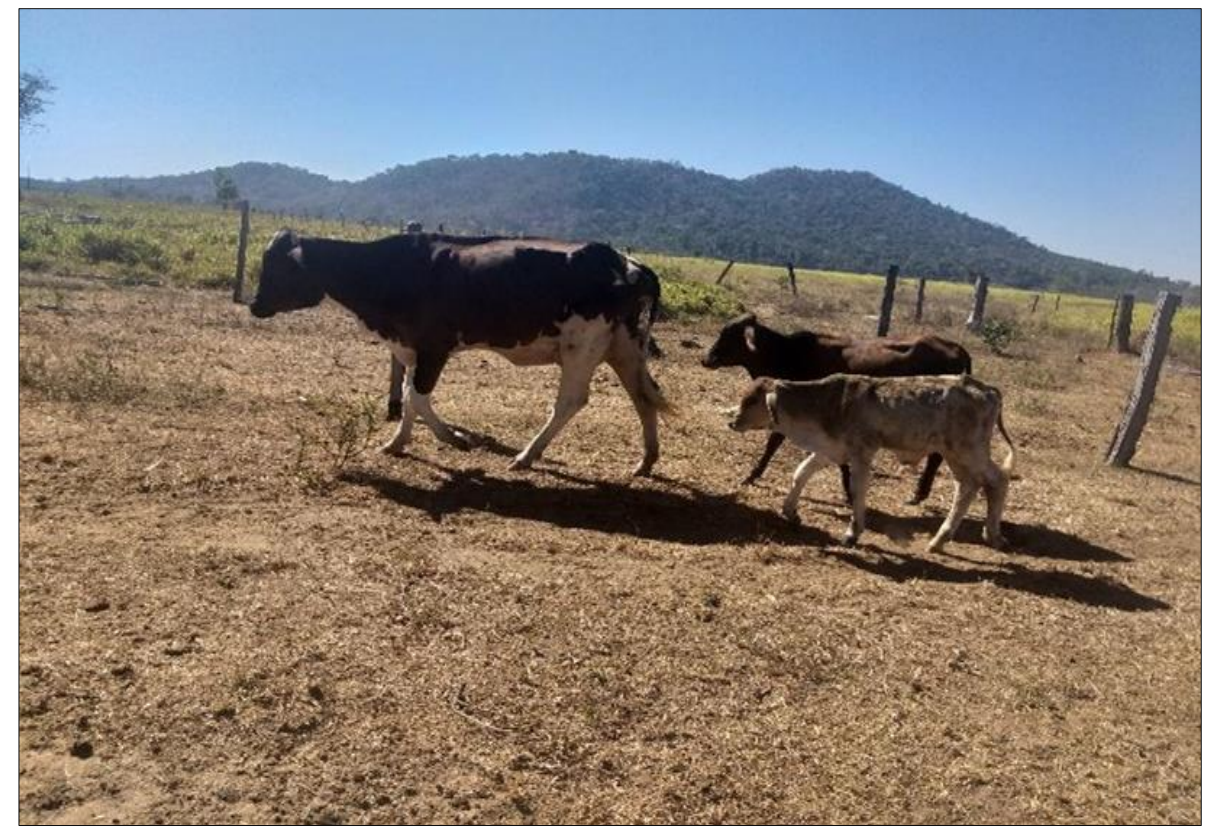

Fonte: Dados coletados pelos autores (2019).

\section{Resultados e Discussão}

Ao analisar a produtividade e manejo dessas propriedades, verificou-se que o apoio de profissionais qualificados interfere significativamente no incremento da produtividade de leite na propriedade Estância do Ouro, que continua inserida no programa Nosso Leite. Na referida propriedade, houve melhora significativa em relação ao Sítio Laço do Ouro cujo proprietário abandonou o projeto. Mesmo a diferença no tamanho das propriedades sendo de apenas 04 (quatro) ha, a propriedade menor (Estância do Ouro) produziu mais que o triplo de leite 
comparando-se com a propriedade maior (Laço do Ouro), conforme verifica-se na Tabela 2.

Tabela 2 - Produção de leite nas propriedades Estância do Ouro (Canabrava do Norte/MT) e Sótio Laço do Ouro (Vila Rica/MT). Fonte: Obtido pelos autores (2019).

\begin{tabular}{ccc}
\hline \multirow{2}{*}{ Descrição } & \multicolumn{2}{c}{ Propriedades } \\
& Estância do Ouro & Sítio Laço do Ouro \\
\hline Tamanho das propriedades (ha) & 48 & 52 \\
Produção Diária (L) & 340 & 96 \\
Preço do litro de Leite $(\mathrm{R} \$)$ & 1,21 & 1,04 \\
\hline
\end{tabular}

A propriedade Laço do Ouro produz atualmente 96 (noventa e seis) litros de leite, tirando manualmente uma vez ao dia, e a Estância do Ouro produz 340 (trezentos e quarenta), sendo que são realizadas duas ordenhas, usando ordenhadeira mecânica. Ainda assim, cabe salientar que a propriedade Estância do Ouro produz mais leite, quase o dobro se for considerada apenas uma ordenha (170 litros de leite).

$\mathrm{Na}$ Tabela 2 também se observa o valor pago no litro de leite, lembrando que esses valores variam de região para região, ou seja, não há rigor no valor estabelecido pelos laticínios que compram o leite diretamente dos produtores. $\mathrm{O}$ valor que estão recebendo com a venda do leite produzido em suas propriedades também afeta o estímulo destes produtores. No presente estudo, enquanto na propriedade Estância de Ouro (Canabrava do Norte/MT) é pago o valor de R\$ 1,21 na propriedade Laço do Ouro (Vila Rica/MT) é pago R\$ 1,04.

Verificou-se, também, que o manejo adequado na nutrição no período de seca, feito por meio de suplementos, mantém a mesma quantidade de leite produzido bem como a condição ideal de ECC dos animais. Observou-se a importância do intervalo entre partos, pois quando realizado de maneira correta este método pode aumentar tanto a produção de animais na propriedade quanto a lucratividade do produtor. Essa prática consiste em retirar a mamada dos bezerros para aumentar a quantidade de leite e quando realizada corretamente tende a aumentar o número de partos.

A gestão da propriedade Estância do Ouro se mostra mais eficiente do que a administração do Sítio Laço do Ouro. Por outro lado, há a necessidade de um trabalho conjunto, entre produtores e técnicos qualificados, objetivando a melhora na produtividade. Por sua vez, o produtor deve ser paciente e persistente, pois o retorno financeiro nestes processos exige tempo, estudo e dedicação para que os resultados positivos apareçam e permaneçam melhorando a produtividade do rebanho leiteiro. 


\section{Considerações}

O presente estudo buscou entender e diagnosticar os métodos de manejo com gado leiteiro com vistas à compreensão dos resultados de produtores rurais adeptos ao Programa Nosso Leite (Embrapa). Assim, denota-se que a propriedade Estância do Ouro (Canabrava do Norte - MT), onde foi mantida a metodologia deste Programa, obteve maior êxito na produtividade de leite do que o Sítio Laço do Ouro (Vila Rica-MT), na qual houve desistência do produtor em seguir os métodos propostos.

Verificou-se que a persistência e a dedicação, a longo prazo, resultou em ganhos significativos ao produtor que manteve a avaliação contanste do manejo da sua produção. $\mathrm{O}$ programa Nosso Leite é de suma importância para o desenvolvimento regional e os produtores, podem comprovar com resultados positivos se forem seguidas as normativas descritas no quadro reprodutivo.

\section{Referências}

BERGAMASCHI, M. A. C. M.; MACHADO, R.; BARBOSA, R. T. Eficiência reprodutiva das vacas leiteiras. Circular Técnica, n. 64. Embrapa Pecuária Sudeste, São Carlos/SP, 2010.

CERDÓTES, L.; RESTLE, J.; ALVES FILHO, D.C.; NÖRNBERG, M. F. B.; NÖRNBERG, J. L.; HECK, I.; SILVEIRA, M. F. Produção e composição do leite de vacas de quatro grupos genéticos submetidas a dois manejos alimentares no período de lactação. Revista Brasileira de Zootecnia, v.33, n.3, p.610-622, 2004.

CRUZ, G. R. B. RIBEIRO, M. N.; PIMENTA FILHO, E. C. Estimativas de parâmetros de curva de lactação de bovinos. Archivos de Zootecnia, v. 58, n. 224, p. 695-704, 2009.

FERREIRA, A. M.; MIRANDA, J. E. C. Medidas de eficiência da atividade leiteira: índices zootécnicos para rebanhos leiteiros. Comunicado Técnico, n. 54. Embrapa Gado de Leite, Juiz de Fora/MG, 2007.

GOMES, T. B. B. Diagnóstico de índices zootécnicos e produtivos do rebanho leiteiro do Departamento de Zootecnia/CCA/UFPB. Centro de Ciências Agrárias (Trabalho de Conclusão de Curso - TCC, Graduação em Zootecnia. Areia/PB. 2018. Disponível em: $<$ https://repositorio.ufpb.br/jspui/handle/123456789/3453>. Acesso em: 07 de agosto de 2019.

IBGE. Instituto Brasileiro de Geografia e Estatística. 2019. Pesquisa trimestral do leite. Disponível em: <https://www.ibge.gov.br/estatisticas/economicas/agricultura-e- 
pecuaria/9209-pesquisa-trimestral-do-leite.html?=\&t=series-historicas $>$. Acesso aos $15 \mathrm{de}$ agosto de 2019.

LAGO, E. P.; PIRES, A. V.; SUSIN, I.; FARIA, V. P.; LAGO, L. A. Efeito da Condição Corporal ao Parto sobre Alguns Parâmetros do Metabolismo Energético, Produção de Leite e Incidência de Doenças no Pós-Parto de Vacas Leiteiras. Revista Brasileira de Zootecnia, v.30, n. 5, p. 1544-1549, 2001. DOI: 10.1590/S1516-35982001000600023

ROSSO, G.; RODRIGUEZ, N. Gestão do rebanho leiteiro ganha aplicativo. 2016.

Embrapa Pecuária Sudeste e Embrapa Informática Agropecuária. Publicado online aos 23 de agosto de 2016. Disponível em: <https://www.embrapa.br/en/busca-de-

noticias?p_p_id=buscanoticia_WAR_pcebusca6_1 portlet\&p_p_lifecycle=0\&p_p_state=pop_ up\&p_p_mode=view\&p_p_col_id=column-

1\&p_p_col_count=1\&_buscanoticia_WAR_pcebusca6_1portlet_groupId=1355045\&_buscan oticia_WAR_pcebusca6_1portlet_articleId=15562958\&_buscanoticia_WAR_pcebusca6_1po rtlet_viewMode=print $>$. Acesso aos 10 de agosto de 2019.

VILELA, D.; FERREIRA, R. P.; FERNANDES. E. N.; JUNTOLLI, F. V. (Eds.). Pecuária de leite no Brasil: cenários e avanços tecnológicos. Brasília/DF: Embrapa, 2016. 435 p. ISBN 978-85-7035-644-4 